\title{
Les voyages du « Moi » et le complexe de la maison, ou le discours de la nostalgie
}

Henri Bonnet

\section{(2) OpenEdition}

12 Journals

Édition électronique

URL : http://journals.openedition.org/recherchestravaux/185

DOI : 10.4000/recherchestravaux.185

ISSN : 1969-6434

Éditeur

UGA Éditions/Université Grenoble Alpes

\section{Édition imprimée}

Date de publication : 15 avril 2007

Pagination : 79-94

ISBN : 978-2-84310-107-7

ISSN : 0151-1874

\section{Référence électronique}

Henri Bonnet, "Les voyages du « Moi » et le complexe de la maison, ou le discours de la nostalgie », Recherches \& Travaux [En ligne], 70 | 2007, mis en ligne le 28 novembre 2008, consulté le 08 septembre 2020. URL : http://journals.openedition.org/recherchestravaux/185 ; DOI : https://doi.org/10.4000/ recherchestravaux.185 
Henri BONNET, Inspecteur général honoraire

\section{Les voyages du «Moi» et le complexe de la maison, ou le discours de la nostalgie}

Depuis la publication en I97I par Georges Lubin de sa remarquable édition, dans la Bibliothèque de la Pléiade, des Lettres d'un voyageur, et la même année, à un moindre niveau, de la petite édition GF qui a été reprise en 2004, bicentenaire oblige, avec une actualisation limitée à la bibliographie, il y a eu un certain nombre d'articles ou de chapitres de qualité qui leur ont été consacrés ${ }^{\mathrm{I}}$.

En m'aventurant dans les pages qui suivent sur les deux routes des voyages $\mathrm{du}$ " moi » et du complexe de la maison, j'ai l'impression de (re)prendre un itinéraire à la fois naïf et obligé qui me fournira les deux premiers aspects de mon questionnement : d'une part le moi, le monde et la bibliothèque ${ }^{2}$, d'autre part le bonheur de la maison, pour appeler d'ores et déjà le " complexe" par son nom.

Je réserve pour un nécessaire troisième temps l'aboutissement de ma démarche que j'appellerai le mystère de « la chambre haute » ou, plus globalement, le discours de la nostalgie. En arrière-fond il y aura parfois l'esquisse d'un rapprochement avec la poétique nervalienne, comme j'avais essayé de l'entreprendre dès $1969^{3}$.

I. Voir la bibliographie en fin de volume.

2. Par référence et hommage au titre de l'ouvrage de Ch. Montalbetti, Le Voyage, le monde et la bibliothèque, Paris, PUF, 1997.

3. H. Bonnet, "George Sand et Gérard de Nerval ", Hommage à George Sand, L. Cellier (dir.), Paris, PUF, 1969, p. II5-I50. 


\section{Le moi, le monde et la bibliothèque}

Sans vouloir remettre mes pas dans ceux de Pierre Laforgue lorsqu'il parle d'" identité bohémienne ${ }^{4}$ ", je voudrais néanmoins apporter une petite contribution à la réflexion sur le bohémianisme du narrateur-héros des Lettres. D'une part il faut préciser que ces voyages commencent au plus près du « moi » et même à l'intérieur de celui-ci : le caravansérail central, comme Max Jacob a parlé de son " laboratoire central ". Je n'en veux pour exemple que sa " descente » dans les enfers de sa personnalité, dans la lettre IV, adressée à l'ami Jules Néraud, figure symbolique du voyage dans les Lettres d'un voyageur. Il y constate et fait apparaitre, pour ne pas dire exploser, le mélange détonant qu'il sent en lui-même : le bien, le mal, les devoirs, "la vertu [comme] habitude », « la foi [...] lueur lointaine [...] dans les espaces du ciel », « la volonté " qui se laisse abuser par « un troisième conseiller plus fort que la foi et que la vertu, l'orgueil » (743-744; p. I29-I3I). Comment et où se diriger dans une page comme celle-ci qui est un vrai tohu-bohu? "Oh, que ma quille éclate! oh ! que j'aille à la mer! ", a-t-on envie de lire.

Le narrateur-héros ne semble que trop savoir où il a envie - lui - d'aller, au plus loin de lui-même et des autres, lorsque, pour échapper à l'emprise d'Éverard, il se fixe un habillement et un itinéraire virtuels : " espèce de garçon de charrue, coiffé d'un chapeau de jonc, vêtu d'une blouse de roulier, chaussé de bas bleus et de souliers ferrés ", il se voit et se veut partant pour Stamboul (lettre VI ; p. 793 ; p. 175) ; il passe par Genève, achète un âne pour traverser les montagnes avec son bagage, remonte la Forêt-Noire pour chercher une plante, visite " un ami islamite " à Corfou, achète à Alexandrie une pipe pour son ami Duteil, pousse pour la femme de celui-ci jusqu'à Alep afin de lui rapporter un châle et un éventail (lettre VI ; p. 816 ; p. 196-197). Ouf ! Quel programme, à faire pâlir Nerval et les autres voyageurs du XIXe siècle.

4. P. Laforgue, Corambé. Identité et fiction de soi chez George Sand, op. cit., p. 75. Je ne tenterai même pas de résumer le stimulant essai de Pierre Laforgue ou d'en donner des idées, tant les idées et les formules fusent sous sa plume, depuis le moment où il distingue fortement (trop, à mon sens) l'auteur du narrateur-héros et fait de celui-ci (à nuancer là aussi) un " personnage inventé de toutes pièces ", jusqu'au moment où il distribue le texte des Lettres dans les " quatre éléments de la fantaisie, du voyage, du bohémianisme et du bariolé " (p. 85). Devant un public qui n’y était pas préparé, je n'ai pas eu de peine à les retrouver dans l'épisode adventice et superbe de la descente, en plein Bugey, aux ruines de la Chartreuse de Mériat, avant le débouché, avec la perspective du mont Blanc, sur le "tapis bigarré " du pays de Gex (lettre X ; p. 893-895 ; p. 27I-273 ; selon la convention que nous adoptons pour ce volume : G. Sand, Lettres d'un voyageur, pages de l'édition de G. Lubin puis pages de l'édition de H. Bonnet). 
De l'intérieur de lui-même au plus extérieur, le «moi » affronte le monde et, troisième dimension, le confronte à une expérience qui est à mi-chemin de l'intérieur et de l'extérieur, celle de la bibliothèque. C'est un point sur lequel, jusque-là, on n'a pas assez insisté à propos de George Sand, alors que, pour Nerval (puisque j'ai choisi de jeter quelques passerelles entre eux), on accumule sur et dans sa tête de voyageur une pratique livresque qui menace toujours de l'écraser.

Le moment où le moi de la bibliothèque commence à s'affirmer dans les Lettres, même s'il n'a pas de rapport direct avec le voyage proprement dit, c'est dans la partie de la lettre IV adressée au Malgache (qui vient après un passage par Rollinat) : le Voyageur, alors à Nohant, se donne comme lecteur de trois ouvrages, lui qui, depuis trois ans, n'a pas lu « la valeur d'un volume in-octavo ». Entre les trois titres cités, L'Eucharistie de l'abbé Gerbet, Réflexions sur le suicide par $\mathrm{M}^{\mathrm{me}}$ de Staël et la Vie de Victor Alfieri par Victor Alfieri, il n'y a pas d'autre rapport que celui, métaphorique, avec le voyage du " moi ». Mais s'il s'étend peu sur L'Eucharistie, les deux autres le touchent davantage, surtout la Vie d'Alfieri qui, "considérée comme livre, est un des plus excellents que je connaisse ", pour sa simplicité, sa froideur de jugement, " une concision et une rapidité pleines d'ordre et de modestie " (p. 752-753; p. I37-I39). Avis aux auteurs d'autobiographie dont elle ne serait peut-être pas. Nous retrouverons, dans les parenthèses de ses errances, une pareille expérience de lecture, portant sur plusieurs jours, avec le temps consacré, dans « la maison déserte ", c'est-à-dire dans la lettre VII, à la lecture de l'Essai de physiognomonie de Lavater dont elle avait déjà pratiqué dans sa jeunesse le dictionnaire portatif. On ne dira jamais assez l'importance de cette lecture dans la vie et l'œuvre de George Sand, et nous aurons l'occasion d'y revenir d'un autre point de vue. Présentement, retenons le rôle de la bibliothèque dans les Lettres. Celle-ci ne saurait se limiter à ces deux gros îlots. Elle s'y retrouve plus souvent en archipels comme, par exemple, dans la lettre IV, Les Natchez, l'abbé Prévost, La Nouvelle Hélö̈se, Goethe et Byron. Quand on remonte dans les lettres vénitiennes, maître Kreisler (donc Hoffmann) est au rendez-vous, mais aussi, dans un autre genre, autre îlot important (précisément dans l'île des Arméniens), Lamennais qui vient d'être condamné pour ses Paroles d'un croyant. Sans vouloir faire un rappel de toutes les allusions livresques, on ne peut passer sous silence la Bible certes, mais aussi, dans le désordre, l'Enéide, Luther, Hamlet, Gozzi, l'Arioste et beaucoup d'autres. Les

5. Telle que j'ai voulu la faire figurer sur la couverture de la réédition GF, en 2004, de mon édition des Lettres d'un voyageur. 
nombreuses allusions mythologiques et historiques qui parsèment le texte viennent aussi de la bibliothèque : Apollon chez Admète, le roi Midas, Prométhée, Marius dans le marais de Minternes, Hercule, Atlas, etc. Comment ne pas penser encore (et surtout ?) que son œuvre à elle, George Sand, a déjà pris place dans la bibliothèque et qu' elle l'accompagne, depuis la chanson d'Engelwald jusqu'à ses romans de la génération de I830 qu'elle défend becs et ongles dans la lettre XII, en passant par Lélia qui lui cause plus d'un souci. Le «moi » du Voyageur, et pas seulement sa livrée, c'est aussi tout ce bagage livresque, qu'il appartienne à autrui ou à elle-même, ce qu'on ne rappelle pas assez souvent.

À l'enseignes des voyages du "moi ", à travers le kaléidoscope des Lettres, avec la très forte nostalgie - premier soulignement - du Tyrol (lettre I), de la "verte Bohême " (lettre VI) et, entre autres, du rêve d'Orient, il me plaît de retenir simplement de George Sand voyageuse et même bohémienne la vignette qu'en fixait Nerval dès I838, la situant entre le "vieux d'Assoucy » et le prince Pückler-Muskau : "George Sand », écrit-il pour justifier moins ses fantaisies que ses propres familiarités, " nous donne bien quelques détails parfois sur sa blouse de forestière, sur sa chaussure éculée ou sur ses maigres soupers, assaisonnés de commis voyageurs ou de larrons présumés dans mainte auberge suspecte ${ }^{6}$ ». George Sand ou l'art de voyager sans apprêt, mais pas sans attrait. Si, dans la lettre à Buloz du 27 mai 18367 , sa figure ne saurait être celle du démon Asmodée, le tentateur biblique, "le maître des amours impures" (selon Georges Lubin), il ne lui déplaît pas de jouer les " oncle Tobie ${ }^{8}$ ", notamment dans la lettre V adressée à François Rollinat, l'ange de la maison si Néraud est celui du voyage.

\section{Le bonheur de la maison}

Avant d'en venir là, c'est-à-dire de franchir un pas important que j'appellerai le pas de Tobie, je voudrais, sinon revenir en arrière, au moins faire apparaittre qu'il y a dans les Lettres d'un voyageur un mouvement qui, au lieu de nous

6. G. de Nerval, repris dans Lorely (I852), chap. "La Forêt-Noire ", in CEuvres complètes, édition de J. Guillaume et Cl. Pichois, Paris, Gallimard «Bibliothèque de la Pléiade ", I9841993, 3 vol., vol. III, p. 2 I.

7. G. Sand, Correspondance, op. cit., vol. III, p. 404-405.

8. Voir la variante de la Revue des Deux Mondes relevée par G. Lubin dans son édition des Lettres d'un voyageur, op. cit., p. I456-I457 (variante $a$ de la p. 760). La référence à Tobie est maintenue dans l'édition en volume (p. 76I; p. I46). 
projeter sur les routes de la vie intérieure comme de la vie extérieure, du «moi " des œuvres comme de celui de la bibliothèque, met en jeu une opération de réintégration : c'est déjà particulièrement sensible, à la fin de la lettre III, lorsque, «à travers les traînes de Torcello " (p. 734 ; p. I20), résonne en elle, quelle que soit la beauté du lieu, l'impérieux désir de rentrer en France. C'est là que la nostalgie commence à redoubler, donc à jouer pleinement son rôle, comme mal du retour consécutif à celui des départs et des errances. Dans son roman L'Ignorance, Milan Kundera la définit très bien : "Sous [l']éclairage étymologique, la nostalgie apparaît comme la souffrance de l'ignorance. Tu es loin et je ne sais pas ce que tu deviens. Mon pays est loin et je ne sais pas ce qui s'y passe9 ".

Sous l'éclairage nervalien, avant même d'aborder ce qu'il en est dans les Lettres d'un voyageur, on se rappellera qu'un des premiers textes de Nerval $\left(\mathrm{I} 83 \mathrm{I}^{\mathrm{I}}\right)$, qui en fait endosser la paternité à Jean-Paul Richter en le présentant comme une traduction ${ }^{\text {II }}$, s'intitule Le Bonheur de la maison, titre que nous reprenons ici pour l'appliquer à George Sand dans ses Lettres. Il serait riche de signification que l'écrivain, que son ami Gautier présente comme un « martinet des tours qui est apode et dont la vie est un vol perpétuel », n’aurait rien eu de plus primordial que de vouloir connaître le bonheur de la maison dans un presbytère de campagne en Allemagne auprès d'un oncle pasteur et d'une jeune Maria dont le destin a été ébranlé à partir du moment où la famille a été dispersée. Un des derniers textes de Nerval, Promenades et souvenirs, se présente comme la recherche d'une maison et finira, au moins pour un temps, dans une roulotte, sinon de bohémiens, au moins de comédiens. N'y a-t-il pas là plus d'une affinité avec la course du voyageur-héros des Lettres?

Certes le voyage, fictif ou réel, ou contaminé des deux, imposé ou volontaire, l'emporte (aux deux sens du mot) au gré des vents et des circonstances. Mais n'y a-t-il pas une voix plus profonde qui l'amènerait à se fixer à Nohant ou dans la demeure que, avec ses tombes (à ne pas oublier ou abandonner), Nohant symbolise ? Les Lettres sont faites du dialogue de la maison et de la route comme, dans Les Maîtres sonneurs, il y a le dialogue entre le Berry et le Bourbonnais, la plaine et la forêt, avec un clin d'œil du côté du pays de L'Astrée qui n'est pas tout à fait à écarter de leur horizon. Il est paradoxal de faire une telle place à " la " maison. C'est le moment où le voyage l'emmène loin d'elle, donc la fait d'autant plus regretter ou désirer ; c'est le moment où

9. M. Kundera, L'Ignorance, Paris, Gallimard, 2003, p. I4.

Io. C'est l'année de Rose et Blanche ou la comédienne et la religieuse.

II. Alors que Claude Pichois a démontré de façon convaincante que ce texte était certainement de Nerval lui-même : Euvres complètes, op. cit., vol. I, p. 314-318 et p. I616-16I7. 
s'offrent au Voyageur d'autres résidences et, surtout, où la maison est au cœur de ses ennuis familiaux et judiciaires. J'ai toujours pensé que le cœur des Lettres, au moins dans l'édition en volume, était les lettres IV et IX, avec, entre les deux, la belle lettre V où George Sand joue le "vieux oncle » avant de résister aux assauts d'Éverard et de partir (en esprit) en direction de la Bohême ${ }^{12}$, au lieu de se laisser mettre le grappin dessus. J'en suis même à penser que le mythe de la maison donne à l'ensemble des Lettres sa structure que j'ai quelque peine à laisser à l'inspiration bohémienne du tout venant et du hasard des circonstances. Pourquoi, comme dans un dessin d'architecte, ne pas faire correspondre aux quatre premières lettres les quatre dernières ? La première aile du bâtiment déboucherait sur la lettre IV à Jules Néraud et l'autre repartirait de la lettre au Malgache, ces deux lettres cardinales (qui d'abord n'en faisaient qu'une dans la Revue des Deux Mondes) pivotant sur la maison de Nohant tour à tour recherchée, trouvée, perdue, retrouvée. Quant au corps central, on aurait en appui facial, de part et d'autre, les lettres $\mathrm{V}$ (du " vieux oncle " Tobie) et VIII (du vieux pervers Talleyrand) et, au centre, comme une sorte de perron, les lettres VI (à Éverard) et VII (à Liszt) qui sont celles des choix définitifs entre les vocations, la lettre à Liszt impliquant une autre maison (" déserte ») que la maison familiale, celle d'une famille d'esprits proches du sien, par Lavater interposé.

Depuis Bachelard, la critique s'est surtout intéressée à ce que pouvait signifier la maison et c'est à cause de lui, dans La Terre et les rêveries du repos, que j'ai parlé du " complexe » de la maison. Il rattache à celui-ci le motif de la grotte et, bien qu'il ne la mentionne pas, je ferai référence, moi, à la grotte d'Oliero de la lettre I. Il le déplace ensuite du côté du labyrinthe : pourquoi n'y inclurait-on pas celui du château de la lettre VIII qui mériterait d'engloutir Talleyrand? Mais, dans une conception positive, voire initiatique, du parcours labyrinthique, comme dans La Comtesse de Rudolstadt, c'est l'ensemble des Lettres qui peut donner l'impression d'émerger à la lumière avec la lettre $\mathrm{X}$ qui est celle de la maison et de la maisonnée retrouvées, avec, à partir de là (au sens strict), la liberté d'esprit aussi bien pour aller à la rencontre de Meyerbeer que pour se retourner contre Nisard. À la suite des travaux de Bachelard et de la revue Circé, c'est à la maison comme refuge que revient Simone Vierne dans George Sand, la femme qui écrivait la nuit: elle y revient à propos de Nohant bien sûr, mais l'étend à « des plages de calme à Paris même » et, surtout, elle fait de « la maison déserte » de la lettre VII, la quin-

I2. Où, probablement, sans qu'elle le sache, elle avait un ancêtre royal, comme le dit Georges Lubin dans l'introduction de son édition des Lettres d'un voyageur, op. cit., p. 644. 
tessence, "la forme la plus achevée de ce refuge ", avec des implications sacrées sur lesquelles je serai amené à revenir quoique un peu autrement. Simone Vierne va, à juste titre, jusqu’à écrire de l'entrée : «il faut porter une main impie " pour passer ce "seuil vénérable " qui a valeur d'" enceinte sacrée ${ }^{13}$ ». N'en était-il pas de même de Nohant, quoique de façon plus familiale, plus familière, plus ouverte aux amis sacro-saints?

Sans rien renier de ces interprétations mais en considérant plus directement le texte, je voudrais attirer l'attention plus particulièrement sur la lettre V. Par rapport aux lettres pivotales IV et IX, adressées pour la plus grande partie à Néraud, l'ami symbole du voyage, la lettre $\mathrm{V}$, dite du vieil oncle à l'origine, consonne bien avec la personnalité de Rollinat, figure totémique de la maison. Je ne dirai surtout pas que c'est celle du bonheur béat : la béatitude n'entre que peu dans le cadre ou dans les cordes de l'âme et de la lyre romantiques. Admirons, au contraire, la complexité (sens dérivé de la notion de complexe) du bonheur qui s'exerce (presque au sens du grec askésis), malgré tout, à ce moment à Nohant et qui, à la fin, plongera dans les affres d'une descente aux enfers, comme l'a qualifié Pierre Laforgue ; George Sand parle, elle, dans cette fin, des « abîmes du désespoir ». Mais, précisément, à l'adresse de l'ami Pylade, quelles diaprures qu'il ne faut pas assimiler aux draperies fréquentes de l'autobiographie romantique! L'ennui, inévitable, y a sa place, avec son cortège de langueur, d'atonie, de fatigue, de malaise, de dégoût (p. 767 ; p. I5I-I52). Mais il ne tient qu'à l'intéressée de le "poétiser ", dit-elle, ou, dirait-on aussi bien, de le "prosaïser ", selon la recette donnée de ce qui peut être un bonheur relatif :

Se vêtir convenablement, selon la saison ; avoir de très bonnes pantoufles, un excellent feu en hiver, un hamac léger en été, un bon cheval au printemps, à l'automne un carré de jardin sablé et planté de renonculiers. (Loc. cit.)

On aboutit esthétiquement à des tableaux flamands ou à des croquis de Téniers, revus et corrigés par Hoffmann. Ou, mieux encore, à des scènes vivantes où les amies et amis tiennent bien leur rôle, prêts à exorciser le mal d'être suivant l'âge ou les goûts :

[...] l'un vieillit en sabots dans son ermitage, ou en robe de chambre dans sa mansarde avec quelques amis; tandis que l'autre encravate et parfume chaque soir une momie qui se donne des airs de vie [...]. (P. 775 ; p. 159.)

I3. S. Vierne, George Sand, la femme qui écrivait la nuit, Clermont-Ferrand, Presses universitaires Blaise-Pascal "Cahiers romantiques, $\mathrm{n}^{\circ} 9$ ", 2004, passim dans le chapitre II, notamment à partir de la p. Ioo. 
On n'est pas seulement à Nohant; à Nohant on ne dira pas que l'hiver («de mon âme ») fait seulement des ravages, puisque la beauté des séquences poétiques ennoblit, voire sublime, les malheurs de l'âme. D'ailleurs, en se reportant au début de la lettre, on verra que les impressions étaient plus mêlées et même, préalablement à l'invocation adressée aux " dieux Lares" (p. $760 ;$ p. I45) de la maison, les chances qui accueillaient « le pèlerin " arrivant «à pied dans la poussière du chemin et dans la brume du soir » étaient de bon augure. C'est ce pèlerin (le mot est important) qui avait préludé par ce beau couplet de reconnaissance :

[...] pourquoi serions-nous tristes, à moins d'être fous ? Nos femmes sont charmantes et nos amis, en est-il de meilleurs ? Est-il beaucoup de mortels qui aient eu dans leur vie le bonheur de réunir sous le même toit, presque tous les jours, $[\ldots]$ douze ou quinze créatures nobles et vraies, et toutes unies entre elles d'une sainte amitié ? (P. 760 ; p. I45.)

Gloire, donc, à l'amitié dans le cadre de la maison, condition d'un bonheur à la fois précaire (adjectif à comprendre aussi au sens du latin precari, prier) et intimement vécu.

Oui, mais l'amitié n'est pas tout, et la jeune George Sand peut rêver d'autre chose, surtout dans la période de crise qu'elle traverse et que la maison peut lui donner de ressentir de façon aiguë. Le vrai bonheur, sous le toit d'une maison comme celle de Nohant, pourrait être conçu autrement : «Et moi, vieux, je suis comme toi ", écrit-elle à Rollinat, « je ne suis l'autre moitié de personne. Il m'importe peu de vieillir, il m'importerait beaucoup de ne pas vieillir seul » (p. 77I ; p. I55). Voilà bien le cri du cœur qui demanderait à s'épanouir dans le bonheur à deux. C'est là que, comme dans la fable de Philémon et Baucis, George Sand a développé sans trop s'étendre celle du bon artiste Watelet et de sa digne épouse (comme aurait dit Homère) Marguerite Le Conte. Elle les a voulus pauvres, contrairement à la vérité historique rétablie par Georges Lubin, elle les a surtout voulus heureux jusqu'à la mort dans leur "maisonnette appelée Moulin-Joli" (p. 771-772 ; p. I56), avatar de Nohant. Il ne faut rien de moins qu'une devise empruntée à Horace pour donner à l'épisode ses lettres de noblesse : «Cur valle permutem Sabina divitias operosiores?" (Pourquoi échangerais-je ma vallée sabine contre le tourment des richesses ?) Traduisons, nous : "Pourquoi échangerais-je ma Vallée Noire et mon refuge de Nohant contre les tracas de la route et, surtout, d'un divorce, donc de la solitude ? "Il me semble capital de considérer l'anecdote du Moulin-Joli comme le récit spéculaire non seulement de la lettre V mais, peut-être, de l'ensemble des Lettres d'un voyageur. On ne peut plus nostalgique, la fable du Moulin-Joli ou le bonheur sans fard et sans phrase, quelque chose qui rappelle la parabole de Tobie et Sara. 
Elle est d'autant plus importante que les Lettres offrent a contrario des contre-modèles, un anti-discours de ce bonheur, donc de la nostalgie. Il est à peine besoin de mentionner - en opposition absolue - la lettre VIII, dite " Le Prince " - où le "il » de la première phrase, l'ami du dialogue, représente précisément François Rollinat. Là, c'est tout l'édifice, le château de Valençay, qui mérite de s'écrouler en engloutissant tout le monde. Ce n'est pas un contre-modèle que constitue le couvent des Arméniens dans l'archipel vénitien : George Sand est trop attachée au " rêve monastique ", comme a dit et montré Jean Pommier, mais les circonstances historiques et plus particulièrement religieuses, liées à la personne et aux tribulations de Lamennais, vicient les conditions d'appréciation, alors que, au moins dans la bibliothèque, «[les] instruments de physique et [le] laboratoire [l']avaient transporté aux temps poétiques de la vie monastique" (lettre III ; p. 720 ; p. I07). Passons, a-t-on envie de dire avec elle. Dans le passage constant et renouvelé que constituent le/les voyage(s) dont les Lettres sont censées rendre compte, ce sont les auberges et les hôtelleries qui fournissent les substituts variables de la maison considérée comme lieu normal de vie. George Sand n'y fait pas beaucoup allusion au début, sinon, par exemple dans la lettre I, au cabaret d'Oliero (p. 67I-672; p. 6I-62), alors qu'il n'est pas question de l'hôtel Danieli de Venise. Mais c'est lorsqu'elle se rend à Genève, après l'issue heureuse de son procès, que là, subitement, les auberges se mettent à exister, au moins littérairement. Il y a, entre autres, l'auberge qui, avant Autun, lui a donné le triste spectacle de patriciens avinés et justifié un sinistre couplet sur les cabarets de village (lettre X ; p. 886-887 ; p. 264-265). La suite du voyage est moins sévère, malgré les commis voyageurs. Il n'empêche que, pour elle comme pour Nerval, selon l'analyse de Françoise Gaillard :

[...] le symbolisme des voyageurs et de l'hôtellerie, lieu de passage et de rencontres fortuites, met en évidence le caractère atomisé de la société - collection d'individus. Le modèle idéal que Nerval oppose à cette réalité, dans d'autres textes oniriques, est celui de la famille, totalité organique et vivante. On ne saurait mieux illustrer la nature même de la société bourgeoise, fondée sur le concept primordial d'individu ${ }^{14}$.

Qu'en déduire pour le compte de George Sand ? Ces conditions, même négatives, font ressortir le havre de paix et de bonheur que méritent ou mériteraient de constituer ou, en tout cas, de symboliser Nohant et certains de ses équivalents au moins provisoires.

I4. F. Gaillard, «Nerval, ou les contradictions du romantisme », Romantisme, n I-2, I97I, p. 133 . 
Le mystère de la « chambre haute » ou le discours de la nostalgie

Diastole, systole, telles sont, par la route et par la maison, les deux phases qui, en se compénétrant, assurent la vie des Lettres, avec, déjà, l'expression d'une double nostalgie. À propos de celle-ci, je reprendrais volontiers l'analyse de ce que l'helléniste Pierre Guillon appelait la nostalgie d'Homère :

[...] le sentiment de la nostalgie, dès qu'il se constitue et se définit, apparaît, selon un schéma quasi hégélien, comme la synthèse de l'un de ces couples de besoins contradictoires qu'on retrouve au fond de l'âme humaine, s'appelant l'un l'autre, par une manière de loi d'équilibre ou de compensation, pareils à ceux que dénombre Simone Weil au début de son Enracinement. Ce sont ici [...] le besoin du retour au bien-être et au repos de l'enfance, et celui de s'élancer plus vite et plus avant à travers le monde et la vie. Voire au-delà de la vie ${ }^{15}$.

La citation de Pierre Guillon me semble bien représenter l'enjeu des Lettres avec leurs deux côtés et, au point de rencontre, l'expression d'une nostalgie qui, d'une part, s'enracine profondément et même pathétiquement dans les couches de l'être et, d'autre part, s'élève à ce que je veux appeler, après George Sand elle-même, "[le mystère de la] chambre haute » (lettre VII ; p. 818 ; p. 200). Ces deux appels - de profondeur et de hauteur - se situent, transposés dans un autre registre, l'un à la fin de la lettre $V$, à laquelle j'ai déjà fait une grande place, l'autre au début de la lettre VII, celle de la maison déserte et de Lavater, à laquelle on ne saurait accorder trop d'importance, le second appel étant pour moi encore plus important que le premier.

Dans la fin de la lettre $V$, sélectionnée en quelque sorte par Pierre Laforgue, "Sand se livre à une terrible description des enfers " que le critique qualifie de "dantesque autant que virgilienn $\mathrm{e}^{16}$ ». Je ne sais pas si je commenterais exactement comme lui ce " passage ", vraiment " passage ", mais c'est bien de l'au-delà de la vie qu'il s'agit, sous l'autorité du modèle romain qu'est Marcus Curtius. Ce patronage, qui n'est pas banal ${ }^{17}$, vient - comme exemple de devotio - de Tite-Live, livre VII de son Histoire romaine, plutôt que de Varron (De lingua latina, livre V), mais celui-ci a le mérite d'être plus bref: « Selon le récit de Procilius, la terre s'ouvrit à cet endroit du forum » appelé désormais lacus Curtius. Des haruspices consultés la « réponse fut que le dieu des Mânes revendiquait un sacrifice expiatoire [...] qu'un certain Curtius ", homme valeureux s'il en était, se hâta de réaliser en se précipitant dans le

I5. P. Guillon, «La nostalgie d'Homère ", Cahiers du Sud, n 325, I954, p. 333.

16. P. Laforgue, Corambé. Identité et fiction de soi chez George Sand, op. cit., p. 8I.

17. Quoiqu'il se rencontre chez Balzac, m'a dit J.-L. Diaz. 
vide, « laissant à sa famille un témoignage durable de son souvenir ». C'est le sacrifice qu'est prêt à faire le Voyageur de la lettre $V$, décidé ou résigné à ne pas repasser le seuil de la maison et à se précipiter dans le Tartare, à l'instar de ceux qui «espèrent en une autre vie, parce qu'ils ont gardé le sentiment du beau éternel et le moyen de le posséder » et veulent " comme Curtius, apaiser la colère céleste et fermer l'abîme derrière eux » (p. 779 ; p. I62). La référence historique traduit à sa façon, infernale, la nostalgie de ce qui pourrait être à la fois voyage et réintégration ou, encore, une sorte de De profundis païen.

Mais, à mon sens, George Sand a rêvé, moins en profondeur qu'en altitude, moins selon un schéma antique que selon une proposition chrétienne, un acheminement vers un type de bonheur que ni le voyage ni la maison, respectivement ou ensemble, ne sont capables de lui donner au maximum.

Au début de la lettre VII, à l'orée de la seconde partie des Lettres (autre structure importante), un "passage " (reprenons le terme) est rarement relevé et emprunté par la critique, celui qui conduit les Apôtres, après la mort et la résurrection du Christ, à se retrouver dans une "chambre haute " (en italique dans le texte) qui, dans les Actes, est située à Jérusalem, « lorsque le prodige de la descente du Paraclet s'accomplit sur les disciples de Jésus » (p. 819; p. 20I). Les déclarations de George Sand ne manquent pas alors d'admiration ni d'élévation. Elle présente la retraite des douze comme " un fait unique dans l'histoire du genre humain " et, à travers leur exemple, comme "incontestable [...] le pouvoir miraculeux de la foi chez l'homme» (p. 8I8-8I9 ; p. 200). On ne peut pas être plus explicite et la nostalgie qu'elle exprime en un discours qu'on peut dire... enflammé, ne laisse pas de doute sur l'espoir qu'elle en attend :

L'homme, redivinisé [c'est elle qui souligne], sortira de cette assemblée, un beau matin de printemps, avec une flamme au front, avec les secrets de la vie et de la mort dans sa main, avec le pouvoir de faire sortir des larmes de charité des entrailles du roc, avec la révélation des langues que parlent les peuples encore inconnus chez nous, mais surtout avec le don de la langue divine perfectionnée, de la musique, veux-je dire, portée à son plus haut degré d'éloquence et de persuasion. (P. 819; p. 200-20I.)

C'est l'ambition de son propre discours que définit ici George Sand.

La retombée (si l'on ose dire) dans la musique peut étonner et même détoner et, adressée à Liszt, le futur auteur des Douze études d'exécution transcendante (1838), même s’il est capable de surprendre "les mystères sacrés", nous ramène à une inspiration humaine après le prodige de la descente du Paraclet : mais n'en a-t-il pas été ainsi pour les Apôtres qui se sont mis à parler en langues? Préalablement, George Sand nous a fait savoir que pour elle " la musique, c'est la prière, c'est la foi, c'est l'amitié » (p. 818 ; p. 199) et que toute réunion d'amis au nom du Christ implique, selon Mathieu, sa présence 
parmi eux. La musique est donc prodige et fait des prodiges. Les Lettres en témoignent, comme s'il s'agissait d'un fil d'Ariane, non seulement à propos de Liszt, mais depuis les barcarolles vénitiennes jusqu'aux opéras de Meyerbeer dont elle apprécie "le feu sacré " (même adjectif que pour Liszt) et dont elle vante la "sublimité du grand moi intérieur " (lettre XI ; p. 922 ; p. 299). Et c'est continuellement que, dans les Lettres, à propos de Berlioz, de Beethoven, de Mozart ${ }^{18}$, donc par le truchement de la musique, il y a appel ou recours à une transcendance quasi divine dont le profane ne peut qu'avoir la nostalgie.

C'est continuellement aussi dans les Lettres que, au lieu de céder à la tentation infernale symbolisée (dignement) par Curtius, il y a, par référence au Christ, à la tradition évangélique et apostolique, à diverses images de Dieu, présence d'une transcendance dont le héros-narrateur garde au moins la nostalgie quand il n'en partage pas la conviction. C'est le spiritualisme, plus encore que les découvertes scientifiques ou prétendues telles, vite dépassées par le matérialiste Gall, qui lui fait apprécier la révélation de Lavater. «Ce n'est pas ", écrit-elle à Liszt, dédicataire bien choisi, " par une dissertation sur la physiognomonie que je veux vous engager à lire Lavater, c'est en vous recommandant ce livre comme une œuvre édifiante, éloquente, pleine d'intérêt, d'onction et de charme " (p. 837 ; p. 218). Autoanalyse du discours de Sand ? Elle n'y va pas par quatre chemins : "Si j'étais comme vous en Suisse, je voudrais aller à Zurich, exprès pour recueillir des documents sur la destinée de cet homme évangélique » (p. 84I ; p. 22I). Saint Lavater ? On en oublierait presque l'intérêt scientifique ou, en tout cas, spécifique porté à l'invention de la physiognomonie.

On ne risque pas d'oublier l'intérêt que George Sand porte, elle, dans les Lettres, à la personne et à la doctrine du Christ, jusqu'à la mise en relief, comme dans un tympan de cathédrale, des « beaux vieillards couronnés, qui apparurent de nouveau plus tard à Jean l'apocalyptique, et dont il put ouïr les divins accords parmi les vents de quelque nuit d'orage sur les grèves désertes de son île " (lettre VII ; p. 8I9 ; p. 20I). Faut-il dire que cet intérêt, qui peut prendre, comme on vient de l'entendre, un tour apocalyptique, traverse les Lettres de part en part ? De la lettre I, avec sa prière - bien détachée entre des lignes de points (p. 664-665; p. 54-55) - et sa formule initiale d'allégeance ( La seule puissance à laquelle je croie est celle d'un Dieu juste, mais paternel»), à la justification idéale ou idéalisée, dans la lettre XII, de ce que sont

I8. Voir/écouter en particulier l'interprétation du Dies irae de Mozart par Liszt à l'orgue de la cathédrale de Fribourg (lettre X). 
pour elle non seulement l'amour (" mon utopie, mon rêve, ma poésie "; p. 94I ; p. 317) mais même le mariage " tel que l'a fait Jésus, tel que l'a expliqué saint Paul, tel encore, si vous voulez, que le chapitre VI du titre V du Code civil en exprime les devoirs réciproques " (loc. cit.). Dans le début de la lettre $\mathrm{V}$, la figure de Tobie, appliquée à " l'oncle » dans un passage omis en $1837^{19}$ mais aussi bel et bien gardée dans un autre, peut nous sembler, au plus profond du désespoir, avoir une fonction de passeur ou de sauveur, mais être aussi une préfiguration du Christ. Dans la fin de la même lettre, c'est bien de Dieu qu'elle se sent personnellement abandonnée : «Dieu n'est plus en moi ", écrit-elle (p. 778 ; p. I6I), comme Nerval dans la seconde partie, chapitre I, d'Aurélia : «[Dieu] n'est plus avec moi $^{20}$ ». En dernier recours, seule la descente aux enfers se présente non comme une échappatoire mais comme un sacrifice expiatoire. Faut-il parler d'un sacrifice christique ? Ainsi la boucle serait bouclée, d'où seul Dieu peut la faire sortir.

Seul Dieu ? Plus modestement et moins égoïstement, le discours de la " nostalgie de la maison de Dieu ${ }^{21}$ ", prend souvent dans les Lettres la forme d'une revendication de justice, en plein accord avec l'une des plus importantes béatitudes évangéliques, mais aussi avec sa situation d'épouse traînée devant les tribunaux, pour la partie centrale, des lettres IV à IX (encore elles !). Même quand elle est moins impliquée personnellement, par exemple à propos du passage de Lamennais chez les Arméniens de l'île Saint-Lazare, sans jouer les redresseurs de torts, elle écrit tout un plaidoyer en faveur de " l'ennemi du pape " (lettre III, p. 72I ; p. IO9), à mi-chemin entre la comédie des moines qui ne veulent pas se compromettre et la tragédie du peuple arménien qui a souffert de la persécution au point que « la neige du mont Ararat en a été rougie jusqu'à la cime où s'arrêta l'arche sainte» (lettre III ; p. $722 ;$ p. I09). Le rêve de justice, peut-être inspiré pour une part de Lamennais et de ses Paroles d'un croyant (I834) et inscrit en lettres de sang sur la neige du mont Ararat, éclate dans la lettre IV sous la forme d'un morceau de prose rythmée, donné - nostalgiquement-comme un griffonnage de ses seize ans et brossant, en strophes menaisiennes, le portrait du juste (p. 748-749; p. I34-I35). «Cette singulière déclaration de mes droits de l'homme ", comme elle l'appelait,

I9. Variante relevée par G. Lubin dans son édition des Lettres d'un voyageur, op. cit., p. I457 (variante $a$ de la p. 760).

20. G. de Nerval, Euvres complètes, op. cit., vol. III, p. 725.

2I. Je reprends le titre, évocateur, du roman d'Hector Bianciotti, Nostalgie de la maison de Dieu, Paris, Gallimard, 2003. George Sand, dans une lettre du 6 décembre I842 adressée à son amie Henriette de La Bigottière, parlera, elle, de "la maison du Christ ", tout en ajoutant qu'elle n'en resterait pas là, car « la maison est trop petite" (Correspondance, op. cit., vol. V, p. 815). 
irrigue - religieusement - la pensée comme la poétique des Lettres. C’est à « la justice ", "éternel rêve des cours simples " (lettre XII ; p. 942 ; p. 318), que, à travers et contre Nisard qui s'était instauré juge malveillant de ses écrits, elle fait appel en dernier ressort, en sa page terminale, comme par un ultime hommage rendu, dans la finitude, à l'infini.

Nostalgiquement et religieusement, "le sauvage amour de la justice " (p. 746 ; p. I32) qui, selon la lettre IV, l'avait amenée à écrire, constitue l'" un des traits de l'âge d'or messianique ${ }^{22}$ ", à situer à la fois dans le passé et dans l'avenir, genèse et apocalypse, au croisement et dans le prolongement des avenues et des avènements du voyage, de la maison et de la "chambre haute ", sous le patronage des trois « $\mathrm{L}$ » : Lamennais, Liszt, Lavater, et avec l'appui latéral des deux « $M$ » : Musset (à gauche) et Meyerbeer (à droite). Sans oublier les amis incontournables, dirait-on aujourd'hui : Néraud et Rollinat et l'ennemi bien contourné : Nisard.

Si j'ai été tenté d'amener les Lettres jusqu'au seuil de la maison de Dieu dont, par des voies détournées, y compris bohémiennes, elles expriment au moins la nostalgie à travers, entre autres, le rêve de la " chambre haute " de Jérusalem, c'est que la cantate à trois voix qu'elles sont finalement pour moila route, la maison, la chambre haute - ne peut pas être désolidarisée des œuvres immédiatement postérieures. Les Lettres vont de I834 à l'édition Bonnaire en volume de 1837 , mais aussi à celle de 1843 chez Perrotin (avec sa préface qui se termine sur la belle allégorie historique de la retraite de Russie et de son soleil de "terre promise "; p. 649 ; p. 40), couvrant un espace d'œuvres majeures qui comprend Spiridion, Les Sept Cordes de la lyre et le lancement de Consuelo. Ces trois œuvres me semblent hautement spirituelles, plus que les Lettres, pour leur seul compte, ne le donneraient à penser. Pour s'en tenir à quelques jalons, n'y a-t-il pas, en elles, quelques prémonitions du couvent de Spiridion, y compris dans la maison déserte, et, encore plus, du culte de l'Esprit dans la spiritualité de la chambre haute ? Le mysticisme des Sept Cordes de la lyre est inscrit en filigrane dans le témoignage rendu par les Lettres, dans et malgré la finitude, à l'infini et à l'ampleur de leur musique dans la variété des douze variations épistolaires. Le destin de la petite Consuelo ne semble-t-il pas sortir tout droit des lettres vénitiennes mais aussi de la place faite à la musique lorsque George Sand dit de celle-ci, à l'adresse

22. Pour reprendre la formule d'Yves Le Hir dans F. de Lamennais, Paroles d'un croyant, édition de Y. Le Hir, Paris, Armand Colin, 1949, p. 76 : "La justice est un des traits de l'âge d'or messianique Psaumes, XLIV, 8, LXXI, 2-3-7 ; Esaïe, XXXII, I6 ; Apocalypse, XIX, II. » 
de Liszt, que " c'est la prière, c'est la foi, c'est l'amitié, c'est l'association par excellence " (p. 8I8; p. 199). Entre les Lettres et ces trois œuvres, la cantate à trois voix que sont les premières prend de l'extension et de la hauteur autour du thème (musical ?) majeur (en majeur ?) que résume et sublime la phrase clé du début (encore !) de la lettre VII : « ce qui est incontestable pour moi, c'est le pouvoir miraculeux de la foi chez l'homme» (p. 819; p. 200).

Qu'en pense George Sand à l'heure des futures Nouvelles Lettres d'un voyageur ou, plus précisément, en I868, c'est-à-dire trente ans plus tard, lorsqu'elle revient dans une sorte d'examen de conscience sur ses Lettres dont elle cite en particulier un extrait de la quatrième ("Eh quoi! ma période de parti pris n'arrivera-t-elle pas ? [...] je suis parfaitement heureux »; p. 755; p. I40), dans la partie adressée à son cher Rollinat (la maison) qui désormais n'est plus ? "J'ai ", dit-elle, " cherché un chemin, je l'ai trouvé, perdu, retrouvé $[\ldots]^{23} »$. Sur ce chemin qui est pour moi celui par excellence de la voyageuse bohémienne, continue de marcher, comme elle écrit, "un vieux ermite [qui] jusqu'à sa dernière heure aura souci de monter ${ }^{24}$ » et non d'en rester à l'embourgeoisement de la maison, comme s'il y avait toujours ou toujours eu en elle une nostalgie de l'élévation spirituelle plus forte que celle de la stagnation matérielle. "Ma seule jouissance ", affirmera-t-elle l'année de sa mort, " est de me maintenir avec les miens dans le chemin qui monte ${ }^{25}$ ».

23. G. Sand, "À propos de botanique ", Nouvelles lettres d'un voyageur : édition de G. Lubin, Paris, Éditions d'aujourd'hui, I977, p. I60 ; édition de E. Sourian, Paris, Des Femmes, 2005 , p. I54.

24. G. Sand, "À propos de botanique ", Nouvelles lettres d'un voyageur, édition de G. Lubin, op. cit., p. I63.

25. G. Sand, Correspondance, op. cit., vol. XXIV, p. 5IO, cité par P. Christophe, George Sand et Jésus, Paris, Éditions du Cerf, 2003, p. I42. 